\title{
Recruitment of Intracavernously Injected Adipose-Derived Stem Cells to the Major Pelvic Ganglion Improves Erectile Function in a Rat Model of Cavernous Nerve Injury
}

\author{
Thomas M. Fandel ${ }^{a, \dagger}$, Maarten Albersen ${ }^{a, b, \dagger}$, Guiting Lin ${ }^{a}$, Xuefeng Qiu ${ }^{a, c}$, Hongxiu Ning ${ }^{a}$, \\ Lia Banie $^{a}$, Tom F. Lue ${ }^{a}$, Ching-Shwun Lin ${ }^{a, *}$ \\ ${ }^{a}$ Knuppe Molecular Urology Laboratory, Department of Urology, School of Medicine, University of California, San Francisco, CA, USA; ${ }^{\mathrm{b}}$ Laboratory of \\ Experimental Urology, Department of Urology, University Hospitals Leuven, Leuven, Belgium; ${ }^{\mathrm{c}}$ Department of Urology, Affiliated Drum Tower Hospital, \\ Nanjing University, School of Medicine, Nanjing, Jiangsu 210008, China
}

\section{Article info}

\section{Article history:}

Accepted July 26, 2011

Published online ahead of

print on August 4, 2011

\section{Keywords:}

Adipose-derived stem cells

Cavernous-nerve injury

Chemokines

Erectile dysfunction

Nerve regeneration

nNOS

Radical prostatectomy nerve

sparing

S-100

SDF-1

Major pelvic ganglion

\begin{abstract}
Background: Intracavernous (IC) injection of stem cells has been shown to ameliorate cavernous-nerve (CN) injury-induced erectile dysfunction (ED). However, the mechanisms of action of adipose-derived stem cells (ADSC) remain unclear.

Objectives: To investigate the mechanism of action and fate of IC injected ADSC in a rat model of $\mathrm{CN}$ crush injury.

Design, setting, and participants: Sprague-Dawley rats $(n=110)$ were randomly divided into five groups. Thirty-five rats underwent sham surgery and IC injection of ADSC $(n=25)$ or vehicle $(n=10)$. Another 75 rats underwent bilateral $C N$ crush injury and were treated with vehicle or ADSC injected either IC or in the dorsal penile perineural space. At $1,3,7(n=5)$, and $28 \mathrm{~d}(n=10)$ postsurgery, penile tissues and major pelvic ganglia (MPG) were harvested for histology. ADSC were labeled with 5-ethynyl2-deoxyuridine (EdU) before treatment. Rats in the 28-d groups were examined for erectile function prior to tissue harvest.

Measurements: IC pressure recording on CN electrostimulation, immunohistochemistry of the penis and the MPG, and number of EdU-positive (EdU+) cells in the injection site and the MPG.

Results and limitations: IC, but not perineural, injection of ADSC resulted in significantly improved erectile function. Significantly more EdU+ ADSC appeared in the MPG of animals with CN injury and IC injection of ADSC compared with those injected perineurally and those in the sham group. One day after crush injury, stromal cell-derived factor-1 (SDF-1) was upregulated in the MPG, providing an incentive for ADSC recruitment toward the MPG. Neuroregeneration was observed in the group that underwent IC injection of ADSC, and IC ADSC treatment had beneficial effects on the smooth muscle/ collagen ratio in the corpus cavernosum.

Conclusions: CN injury upregulates SDF-1 expression in the MPG and thereby attracts intracavernously injected ADSC. At the MPG, ADSC exert neuroregenerative effects on the cell bodies of injured nerves, resulting in enhanced erectile response.

(C) 2011 European Association of Urology. Published by Elsevier B.V. All rights reserved.

\footnotetext{
$\dagger$ These authors contributed equally.

* Corresponding author. Department of Urology, University of California, San Francisco, CA 94143-0738, USA. Tel. +1 415476 3800; Fax: +1 4154763803.

E-mail address: clin@urology.ucsf.edu (C.-S. Lin).
} 


\section{Introduction}

Nerve-sparing radical prostatectomy is the treatment of choice for localized prostate carcinoma in sexually active men [1]. Despite technical and anatomical advances, erectile dysfunction (ED) remains a major complication of this surgery [2]. Phosphodiesterase type 5 inhibitor therapy is a commonly used first-line treatment for ED following bilateral nerve-sparing radical prostatectomy in ad hoc and erectile rehabilitation schemes [3]. However, due to the limitations in nerve regeneration, these drugs remain largely inefficient in this population [4].

Recently, stem cell-based therapy has garnered attention as a potential alternative in the prevention of ED following cavernous nerve $(\mathrm{CN})$ injury. Embryonic stem cells transduced with brain-derived neurotrophic factor and adult bone marrow-derived stem cells were capable of preserving erectile function via intracavernous (IC) application [5-7]. Despite these encouraging results, the clinical applicability of these cell types is limited due to scant availability and ethical concern [8]. In contrast, adipose-derived stem cells (ADSC) have emerged as one of the most promising stem-cell types [9]. ADSC represent an abundant and easily accessible source of stem cells. While stem cells from both sources can be easily expanded in culture, adipose tissue can be obtained in large amounts with a minimal invasive procedure, and this may exclude the need for in vitro expansion. Furthermore, ADSC lack the ethical burden of the use of embryonic material. IC injection of ADSC has been shown efficacious in restoring damaged nerves and improving erectile function in a $\mathrm{CN}$ injury ED rat model [10].

One common feature in the above-mentioned studies is the administration of stem cells through IC injection. However, despite its apparent therapeutic efficacy, the mechanism of action of this stem-cell transplantation approach has never been investigated. This kind of information is essential before future human clinical trials can be initiated [11]. The present study was designed to examine the fate of ADSC after IC injection following $\mathrm{CN}$ injury and to evaluate the mechanisms of action linked to the observed enhancement of erectile function.

\section{Methods}

\subsection{Study design}

A total of 110 male Sprague-Dawley rats (aged $3 \mathrm{mo}$ ) were obtained from Charles River Laboratories (Wilmington, MA, USA). After harvest of paratesticular fat in all animals, 10 random animals served as noninjected sham controls (group 1 ) (Table 1). The remaining 100 rats were randomly divided into four equal groups (Table 1; Fig. 1): IC injection of ADSC without prior CN crush (group 2); IC injection of phosphate-buffered saline (PBS) after bilateral CN crush (group 3); IC injection of ADSC after bilateral $\mathrm{CN}$ crush (group 4); and perineural injection of ADSC around the dorsal nerve following bilateral $\mathrm{CN}$ crush (group 5). In groups $2-5$, five animals were euthanized at $1 \mathrm{~d}, 3 \mathrm{~d}$, and $7 \mathrm{~d}$ postinjection, respectively, and tissues were harvested to determine the distribution of ADSC in the penis and the major pelvic ganglia (MPG). At $28 \mathrm{~d}, 10$ animals in each group were used to determine recovery of

Table 1 - Distribution of rats in various treatment groups

\begin{tabular}{lcccc}
\hline Group & Rats used at $1 \mathrm{~d}, n$ & Rats used at $3 \mathrm{~d}, n$ & Rats used at $7 \mathrm{~d}, n$ & Rats used at $28 \mathrm{~d}, n$ \\
\hline Group 1: Sham control & & & 5 & 10 \\
Group 2: Sham + ADSC & 5 & 5 & 5 & 10 \\
Group 3: Crush control & 5 & 5 & 5 & 10 \\
Group 4: Crush + ADSC & 5 & 5 & 5 & 10 \\
Group 5: Crush + ADSC & 5 & 5 & 10 \\
\hline
\end{tabular}

ADSC = adipose-derived stem cells; Sham control = sham operation without injections; Sham + ADSC = sham operation and intracavernous injection of ADSC; Crush Control = bilateral cavernous nerve crush and intracavernous injection of phosphate-buffered saline; Crush + ADSC = bilateral cavernous nerve crush and intracavernous injection of ADSC; Crush $+\mathrm{ADSC}_{\mathrm{p}}=$ bilateral cavernous nerve crush and perineural injection of $\mathrm{ADSC}$.



Fig. 1 - Diagrams depicting intracavernous (IC) and perineural injections. IC injection was applied to Sham plus adipose-derived stem cell (ADSC), Crush-control, and Crush + ADSC groups. Perineural injection was applied to the Crush plus perineurally injected ADSC (ADSC p $_{\text {) }}$ group. The structures in the diagram represent the dorsal artery (red), the dorsal vein (blue), the dorsal nerve (yellow), the corpus cavernosum (green/red), and the urethra (purple). 
erectile function before tissue harvest. All animal experiments were approved by the institutional animal care and use committee at the University of California, San Francisco.

\subsection{Adipose-derived stem cell isolation and labeling}

ADSC were isolated from paratesticular fat and plated as described earlier [10]. After $5 \mathrm{~d}$ of incubation, cells were labeled with the thymidine analog 5-ethynyl-2-deoxyuridine (EdU) (Invitrogen, Carlsbad, CA, USA) for $48 \mathrm{~h} \mathrm{[12]} \mathrm{and} \mathrm{then} \mathrm{harvested.}$

\subsection{Induction of cavernous-nerve injury and adipose-derived stem cell injection}

Under 2\% isoflurane anesthesia, the $\mathrm{CN}$ and MPG were identified posterolaterally on both sides of the prostate [13]. In group 2 (Sham + ADSC), no further manipulation was performed. In groups $3-5$, the $\mathrm{CN}$ was isolated and crushed for $2 \mathrm{~min}$ per side at $5 \mathrm{~mm}$ from its origin in the MPG using a dedicated needle holder. Next, the penis was exposed and each rat received an injection of $0.2 \mathrm{ml}$ PBS or 2 million autologous ADSC in $0.2 \mathrm{ml}$ PBS into the corpus cavernosum or the perineural space (Fig. 1). Before injection, an elastic tourniquet was applied at the base of the penis and kept in place for $2 \mathrm{~min}$. A concentration of 2 million cells was the approximate yield of cells after bilateral paratesticular fat harvest and cell-culture expansion for $1 \mathrm{wk}$. This amount falls within the range of cell numbers in previously published studies on cellular therapy for ED $[5-7,10]$. In groups $2-5$, five animals were euthanized at $1 \mathrm{~d}, 3 \mathrm{~d}$, and $7 \mathrm{~d}$ postinjection, respectively, and penile tissue and both MPG were harvested for histology.

\subsection{Measurement of erectile function}

Four weeks after $\mathrm{CN}$ crush or sham surgery, erectile function was assessed. Under ketamine $(100 \mathrm{mg} / \mathrm{kg})$ and midazolam $(5 \mathrm{mg} / \mathrm{kg})$ anesthesia, the MPG and CN were exposed bilaterally via midline laparotomy. A 25-G butterfly needle was inserted into the proximal left corpus cavernosum, filled with $250 \mathrm{U} / \mathrm{ml}$ heparin solution, and connected to a pressure transducer (Utah Medical Products, Midvale, UT, USA) for intracavernous pressure (ICP) measurement. The ICP was recorded at a rate of 10 samples per second. A bipolar stainless-steel hook electrode was used to stimulate the $\mathrm{CN}$ distally from the crush injury site (each pole was $0.2 \mathrm{~mm}$ in diameter, separated by $1 \mathrm{~mm}$ ) via a signal generator (National Instruments, Austin, TX, USA) and a custombuilt constant-current amplifier generating monophasic rectangular pulses with stimulus parameters being $1.5 \mathrm{~mA}, 20 \mathrm{~Hz}$, pulse width of $0.2 \mathrm{~ms}$, and duration of $50 \mathrm{~s}$. Three stimulations were conducted on either side separately, and the maximal amplitude of ICP during nerve electrostimulation was calculated from baseline value and included for statistical analysis of each animal. Systemic blood pressure for the calculation of the ICP increase-to-mean arterial pressure (MAP) ratio was recorded during $\mathrm{CN}$ electrostimulation using a $25-\mathrm{G}$ butterfly needle inserted into the aorta at the level of the iliac bifurcation. After functional testing, animals were euthanized by intraperitoneal injection of pentobarbital $(200 \mathrm{mg} / \mathrm{kg})$ followed by bilateral thoracotomy. The penis and the MPG were then harvested for histologic analysis.

\subsection{Histology}

2.5.1. Immunofluorescence and immunochemistry

Freshly dissected tissue was fixed and cryoembedded [14]. Sections were cut at $5 \mu \mathrm{m}$ and incubated with $3 \%$ goat serum/PBS/0.3\% triton X-100. Tissues were incubated overnight at room temperature with rabbit antineuronal nitric oxide synthase (nNOS) antibody (Santa Cruz
Biotechnology, Santa Cruz, CA, USA); rabbit antistromal cell-derived factor 1 (SDF-1) antibody, a chemokine (Santa Cruz Biotechnology); or mouse anti-S100 (S-100 protein) antibody, a glial-cell marker (Chemicon, Temecula, CA, USA), followed by 1-h immersion in 1:500 dilution of secondary antibody conjugated with Alexa-488 Fluor (Invitrogen, Carlsbad, CA, USA). To visualize ADSC, slides were incubated with freshly made Click-iT reaction cocktail (Invitrogen), which contained Alexa-594 Fluor, for $30 \mathrm{~min}$ at room temperature [12]. Nuclear staining was performed with 4',6-diamidino-2-phenylindole (DAPI; Invitrogen). Slides were stained according to Masson's trichrome-staining protocol for connective tissue and smooth muscle histology.

\subsubsection{Image analysis}

Computerized histomorphometric analysis was performed as described earlier [13]. To quantify EdU staining, penile tissues and MPG were analyzed at $\times 100$ magnification and the number of EdU-positive nuclei per field was recorded. For the analysis of nNOS content, the area of nNOS-positive fibers in the dorsal nerves and the total area of the dorsal nerves were calculated at $\times 400$ magnification. Similarly, SDF- 1 expression in the MPG and the total MPG area were calculated at $\times 100$ magnification. Smooth muscle-to-collagen ratio was determined on the total cavernosal tissue area at $\times 100$ magnification.

\subsection{Statistical analysis}

Results were analyzed using Prism 4 (GraphPad Software, San Diego, CA, USA) and expressed as mean plus or minus standard error of the mean. Multiple groups were compared using one-way analysis of variance followed by the Tukey-Kramer test for post hoc comparisons. Two-group comparison was done using the unpaired $t$ test. Statistical significance was set at $p<0.05$.

\section{Results}

\subsection{Intracavernous but not perineural injection of adipose-} derived stem cells, prevents cavernous nerve injury-induced erectile dysfunction

Erectile function was assessed by electrostimulating the distal end of $\mathrm{CN}$ at $28 \mathrm{~d}$ postsurgery in all groups (Table 2; Fig. 2). Sham-treated rats with or without IC injection of ADSC displayed normal ICP curves and the ICP-to-MAP ratios did not differ significantly (data not shown). $\mathrm{CN}$ crush consistently resulted in significantly decreased ICP-to-MAP ratios. ED was ameliorated by IC injection of ADSC, as rats in the Crush + ADSC group showed significantly improved ICPto-MAP ratios. However, perineural injection of ADSC had no significant treatment effect in $\mathrm{CN}$-injured rats. MAP did not differ significantly among groups.

\subsection{Intracavernous injection of adipose-derived stem cells improves the smooth muscle-to-collagen ratio in the corpus cavernosum}

The corpus cavernosum was examined for the content of smooth muscle and collagen on slides stained with Masson's trichrome (Fig. 3). In sham-operated rats, the smooth muscle-to-collagen ratio was $9.4 \pm 1.3$. In penile tissues of rats that received IC ADSC, the smooth muscle-tocollagen ratio $(9.4 \pm 1.7)$ was almost fully preserved and significantly higher than in the vehicle group $(5.2 \pm 0.8)$ or the 
Table 2 - Intracavernous and mean arterial pressure measurements on cavernous nerve electrostimulation

\begin{tabular}{lccc}
\hline Group & $\begin{array}{c}\text { Maximum ICP, } \\
\mathrm{cm} \mathrm{H}_{2} \mathrm{O}\end{array}$ & $\begin{array}{c}\text { Change in ICP } \\
\text { from baseline }\end{array}$ & $\begin{array}{c}\text { Change in } \\
\text { ICP/MAP }\end{array}$ \\
\hline Sham + ADSC & $164.4 \pm 7.1^{*}$ & $136.9 \pm 4.3^{*}$ & $0.92 \pm 4.3^{*}$ \\
Crush control & $42.5 \pm 5.3$ & $20.8 \pm 4.2$ & $0.13 \pm 2.0$ \\
Crush + ADSC & $154.5 \pm 18.8^{*}$ & $116.0 \pm 18.5^{*}$ & $0.68 \pm 11.1^{*}$ \\
Crush + ADSC p $_{\mathrm{p}}$ & $82.5 \pm 12.7$ & $50.1 \pm 9.9$ & $0.24 \pm 4.1$ \\
\hline
\end{tabular}

ADSC $=$ adipose-derived stem cells; $\mathrm{ICP}=$ intracavernous pressure; $\mathrm{MAP}=$ mean arterial pressure; Sham + ADSC $=$ sham operation and intracavernous injection of ADSC; Crush Control = bilateral cavernous nerve crush and intracavernous injection of phosphate-buffered saline; Crush + ADSC = bilateral cavernous nerve crush and intracavernous injection of ADSC; Crush + ADSCp $=$ bilateral cavernous nerve crush and perineural injection of ADSC.

" $p<0.001$ versus Crush control.

perineurally injected group $(5.7 \pm 0.7)$. There was no significant difference between the vehicle and the perineurally injected groups $(p=0.51)$.

\subsection{Intracavernous injection of adipose-derived stem cells} enhances neuronal nitric oxide synthase-positive nerve regeneration

At $1,3,7$, and $28 \mathrm{~d}$ after $\mathrm{CN}$ crush injury, the level of nNOS expression in the penile dorsal nerves decreased $44 \%, 84 \%$, $76 \%$, and $72 \%$, respectively (Fig. 4). In rats treated with IC ADSC injection, the decreases were $50 \%, 76 \%, 62 \%$, and $40 \%$, respectively. While Crush-Control and Crush + ADSC groups at $1 \mathrm{~d}$ and $3 \mathrm{~d}$ post-CN injury showed similar decline in nNOS expression, the rate of regeneration at $7 \mathrm{~d}$ and $28 \mathrm{~d}$ was significantly different. In particular, the nearly two-fold difference between Crush-Control and Crush + ADSC groups at $28 \mathrm{~d}$ suggests a prolonged neuroregenerative effect of IC ADSC injection.

\subsection{Rapid and time-dependent disappearance of}

intracavernously injected adipose-derived stem cells from the penis

Penile tissues of rats that received IC or perineural injection of ADSC were examined for the presence of ADSC at $1 \mathrm{~d}, 3 \mathrm{~d}$, $7 \mathrm{~d}$, and $28 \mathrm{~d}$ postinjection. The results show that $<50$ ADSC per field $(\times 100$ magnification) were found at each time point in both IC injected groups (Sham + ADSC and Crush + ADSC) (Fig. 5A). In contrast, $>300$ ADSC per field were found in the perineurally injected group (Crush + $\mathrm{ADSC}_{\mathrm{p}}$ ) at day 1 . At day 3 and day 7 the number of ADSC in this group was still significantly higher than in the two IC injected groups. At day 28, no ADSC were detectable in all three groups.

\subsection{Emergence of adipose-derived stem cells in the major pelvic ganglia}

The MPG of rats that received IC or perineural ADSC injection were also examined for the presence of ADSC. The results show that at $1 \mathrm{~d}, 3 \mathrm{~d}$, and $7 \mathrm{~d}$ after treatment, significantly more ADSC were found in the Crush + ADSC group than in either the Sham + ADSC or Crush + ADSC $_{p}$ group (Fig. 5B). At $28 \mathrm{~d}$, few ADSC were identifiable in all three groups.

3.6. Elevated expression of stromal cell-derived factor-1 in the major pelvic ganglia of cavernous nerve-injury rats

To identify possible mechanisms for the appearance of IC injected ADSC in the MPG, the expression of the chemokine
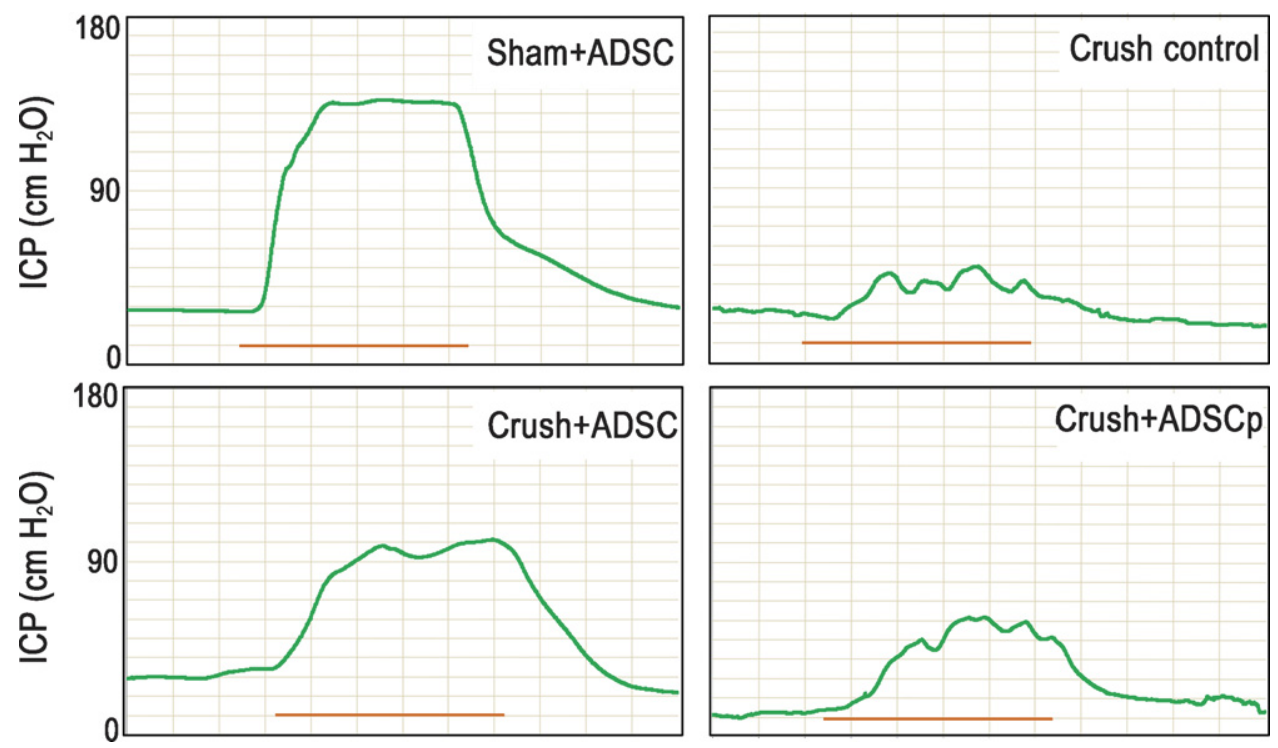

Fig. 2 - Electrostimulation of cavernous nerves after 4 wk. (A) Top: the effects of intracavernous and perineural injection of adipose-derived stem cells (ADSC) on the increase of intracavernous pressure (ICP) on electrostimulation of the cavernous nerve (CN) (each group: $n=10)$. Bottom: ratio of ICP to mean arterial pressure. The red bars are representative ICP recordings of a 50-s electrical stimulation of the CN.

Sham + ADSC = sham operation and IC injection of ADSC; Crush control = bilateral CN crush and intracavernous (IC) injection of phosphate-buffered saline; Crush + ADSC = bilateral CN crush and IC injection of ADSC; Crush + ADSC $_{p}=$ bilateral CN crush and perineural injection of $A D S C$. 



Fig. 3 - Masson's trichrome staining in a penile midshaft specimen. Representative images of the corpus cavernosum in each cohort: (A) Sham plus adipose-derived stem cells (ADSC), (B) Crush control, (C) Crush + ADSC, (D) Crush plus perineurally injected ADSC (ADSC ( $_{\mathrm{p}}$; original magnification: $\times 100$. The graph depicts the ratio of smooth muscle-to-collagen in penile midshaft sections.

Sham + ADSC = sham operation and IC injection of ADSC; Crush control = bilateral CN crush and intracavernous (IC) injection of phosphate-buffered saline; Crush + ADSC = bilateral CN crush and IC injection of ADSC; Crush + ADSC $_{p}=$ bilateral CN crush and perineural injection of $A D S C$.

${ }^{\#} \boldsymbol{p}=<\mathbf{0 . 0 5}$.

SDF-1 was examined. As shown in Fig. 6, SDF-1 expression was significantly elevated in the MPG of $\mathrm{CN}$-injury rats when compared with sham-operated rats. In addition, the transplanted cells appeared to be attracted to the SDF1-expression sites. In Fig. 7, the close proximity of ADSC to MPG nerve fibers is shown at $7 \mathrm{~d}$. ADSC cells did not show signs of engraftment or differentiation into neural tissue types.

\section{Discussion}

Various reports have illustrated the efficacy of stem cells in animal studies of ED $[5-7,10,15,16]$. This study was conducted in a series of experiments aimed at demonstrating efficacy of ADSC in a postprostatectomy model of ED and elucidating the mechanisms of action of this cell-based regenerative therapy. The current study clarifies in detail how ADSC possibly exert their neurotrophic effects.

IC injection was employed for the administration of stem cells in all published studies that investigated their therapeutic potential for ED. In regard to diabetic ED, the IC injected stem cells might exert their effects locally, so as to prevent and/or reverse endothelial damages [15]. However, in the case of $\mathrm{CN}$ injury-induced ED, local interactions in the cavernous tissue are unlikely to have a direct effect on the damaged neurons in the MPG. Of interest, the route of administration appeared to play a major role in establishing the beneficial effects of ADSC therapy in $\mathrm{CN}$-injured rats. The perineural injection was performed based on previous reports identifying retrograde axonal transport of growth factors along the $\mathrm{CN}$ [17]. Furthermore, it was hypothesized that perineural injection 

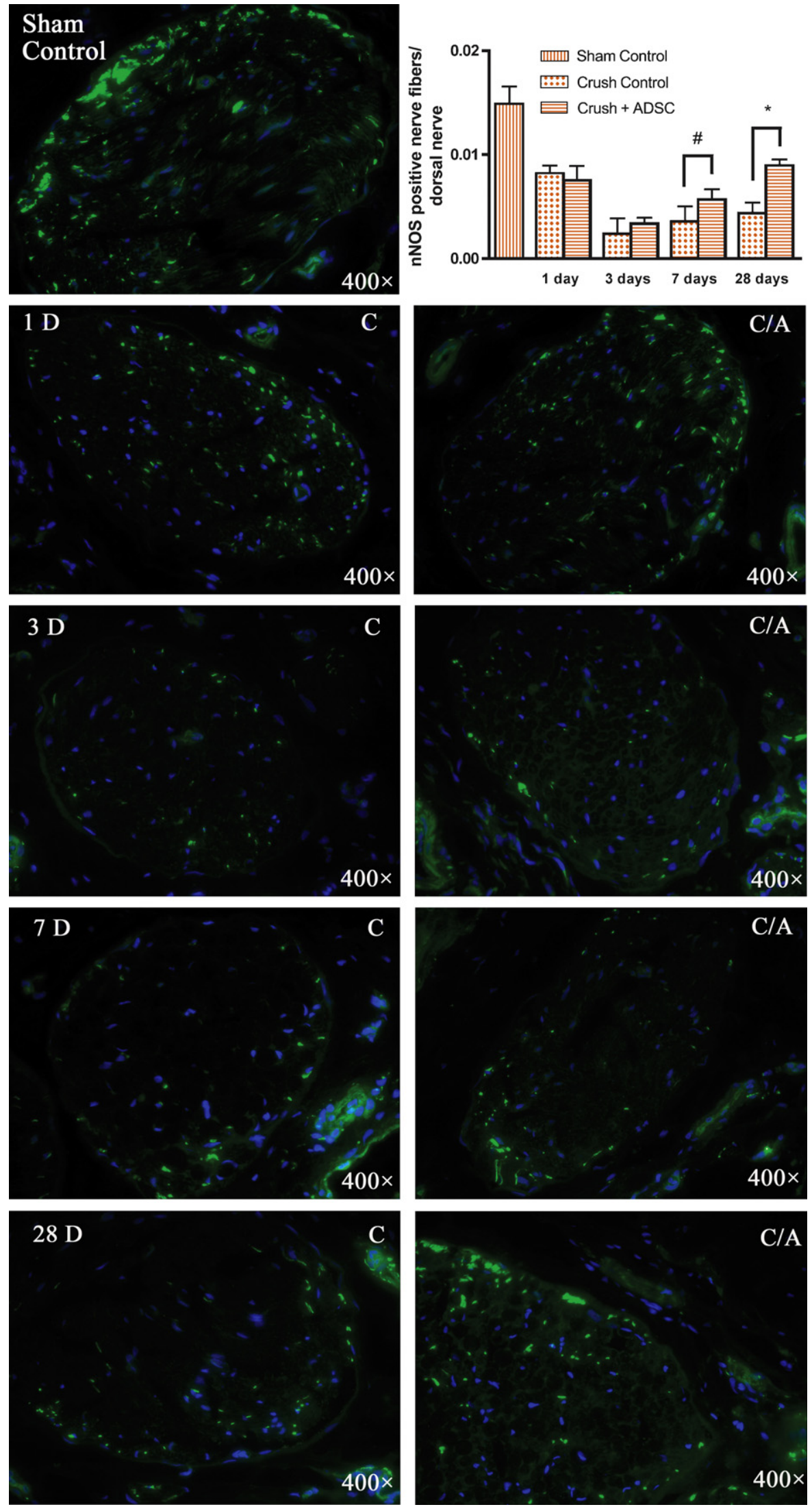

Fig. 4 - Neuronal nitric oxide synthase (nNOS) staining in a penile midshaft specimen. Rats ( $n=5$ in each group) were treated with sham operation (sham control) or bilateral cavernous-nerve (CN) crush, and then intracavernous injection (IC) of adipose-derived stem cells (ADSC) or phosphate-buffered saline (PBS). Tissues were harvested at the indicated time points after injections. Sections were stained for nNOS (green) and 4',6-diamidino-2-phenylindole (DAPI) (blue). Original magnification: $\times \mathbf{4 0 0}$. The graph demonstrates the effect of intracavernous ADSC injection on neuroregeneration in the dorsal nerve over time. 

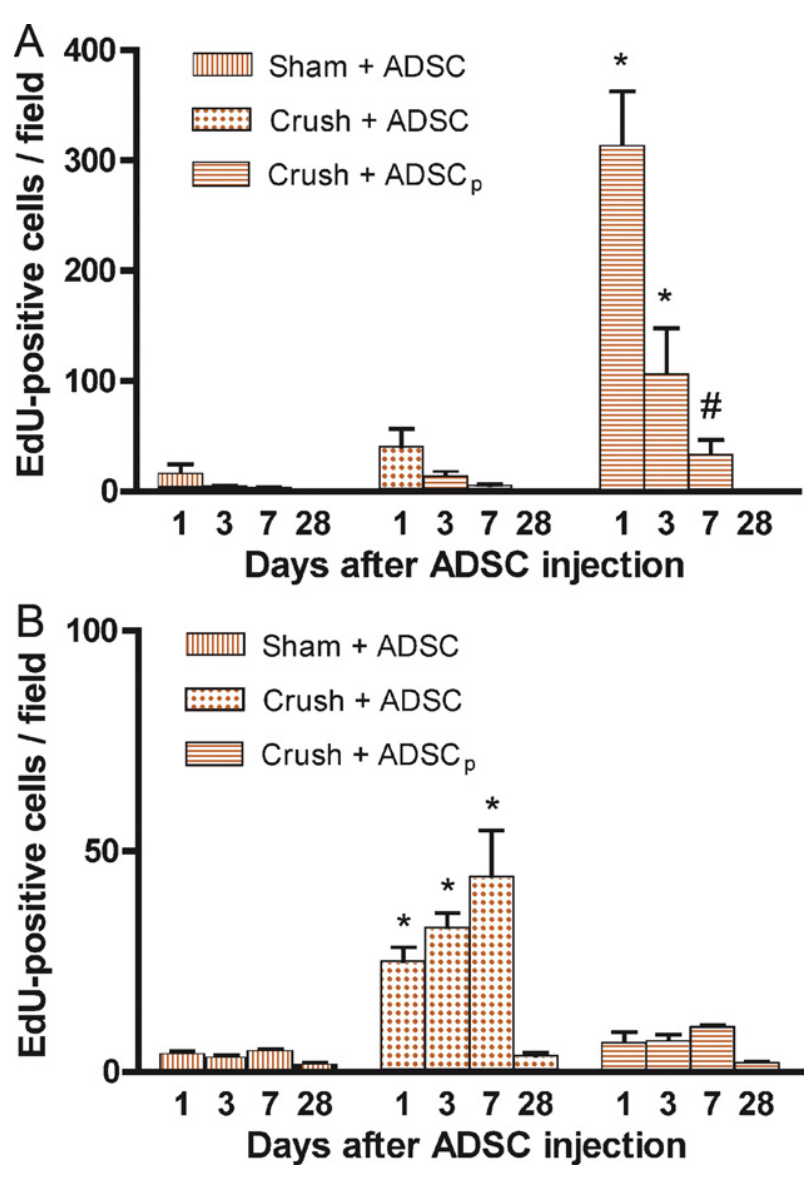

Fig. 5 - Dynamics of 5-ethynyl-2-deoxyuridine (EdU)-positive adiposederived stem cells (ADSC) present in penile tissues and major pelvic ganglia (MPG) over time. Rats ( $n=5$ in each group) were treated with sham operation or bilateral cavernous-nerve (CN) crush and then transplanted with EdU-labeled ADSC either through intracavernous (IC) or perineual injection. (A) Comparison of ADSC numbers in the penile injection site. At the indicated time points, penile tissues were processed for assessment of EdU-labeled cells per field ( $\times 100$ magnification). (B) Comparison of ADSC numbers in the MPG. At the indicated time points, the MPG were processed for the determination of EdU-labeled cells per field $(\times \mathbf{1 0 0}$ magnification).

Sham + ADSC $=$ sham operation and IC injection of ADSC;

Crush + ADSC = bilateral CN crush and IC injection of ADSC;

Crush $+A_{D S C}=$ bilateral $C N$ crush and perineural injection of ADSC. ${ }^{\#} p<0.05$ compared with either of the other two groups at the given time point.

p $<0.001$ compared with either of the other two groups at the given time point.

might allow the injected cells to stay longer in the penis than IC injection because the perineural space is relatively static in terms of blood flow when compared with the cavernous tissue. Surprisingly, in this particular group, erectile recovery was nearly absent. Furthermore, a very limited number of EdU-labeled ADSC appeared in the MPG when cells were injected in a perineural site. In sharp contrast, when the same number of cells was injected in a vascular organ (the corpus cavernosum), a recovery of erectile function to a near-normal status was observed. In addition, ADSC appeared transiently in the MPG a few days after IC injection. From these data it may be concluded that $\mathrm{CN}$ injury appears to provide an incentive for ADSC to migrate to the MPG. To identify this stimulus, we examined SDF-1 expression in the MPG of rats $1 \mathrm{~d}$ after $\mathrm{CN}$ injury. The results show that SDF-1 was detectable in the MPG of $\mathrm{CN}$-injury but not sham-treated rats.

This finding is analogous to previously reported findings in the somatic nerve system of upregulation of SDF-1 in the dorsal root ganglia following sciatic nerve crush for the establishment of neuropathic-pain animal models [18]. ADSC express a number of receptors for a class of small molecules termed chemokines, among which is CXCR4, the receptor for the chemokine SDF-1 [19]. It thus appears that the neuroinflammatory response following nerve injury can be employed to direct regenerative cells toward the neuronal cell body. It is becoming increasingly recognized that neuroglia, such as Schwann and satellite cells, regulate this neuroinflammation, an important process in Wallerian nerve degeneration. This process is coordinated mainly by tumor necrosis factor alpha (TNF- $\alpha$ ), which is strongly upregulated early after nerve injury in the PNS [20]. We have demonstrated how interfering with the TNF- $\alpha$ pathway increases $C N$ regeneration [14]. Neuroglia play a key role in the response to injury by secreting chemokines that attract macrophages and other inflammatory cells, but likely also stem cells, as ADSC express a similar chemokine-receptor panel to macrophages and other subtypes of leukocytes [19,21].

Our previous study demonstrated that IC injection of ADSC ameliorates CN injury-induced ED by preservation of nNOS expression and corpus cavernosum composition [10]. This finding was confirmed in this study; but more importantly, the present study also examined the time course of nerve regeneration. Following $\mathrm{CN}$ crush, a decrease in nNOS expression in the penile nerves was observed over a short time. Following this decrease, nNOS levels started to rise again in all animals, demonstrating a physiologic neuroregenerative response after nerve injury. This process, however, was significantly enhanced by IC injection of ADSC, and was most pronounced after ADSC had started to appear in the MPG. ADSC found in the MPG did not colocalize with neuronal or glial cell markers, ruling out in situ differentiation. Another argument against incorporation and differentiation is that the presence of ADSC in the MPG was transient. Our group previously showed that the ADSC secretome encompasses a variety of neurotrophic signaling molecules such as CXCL5 (the ELR+ CSC chemokine, LIX) [22]. Furthermore, it has been shown that the ADSC secretome in vitro enhances neurite growth [22-24]. Thus, while the precise molecular mechanism remains to be determined, ADSC are capable of enhancing nerve regeneration through the secretion of neurotrophic factors. 


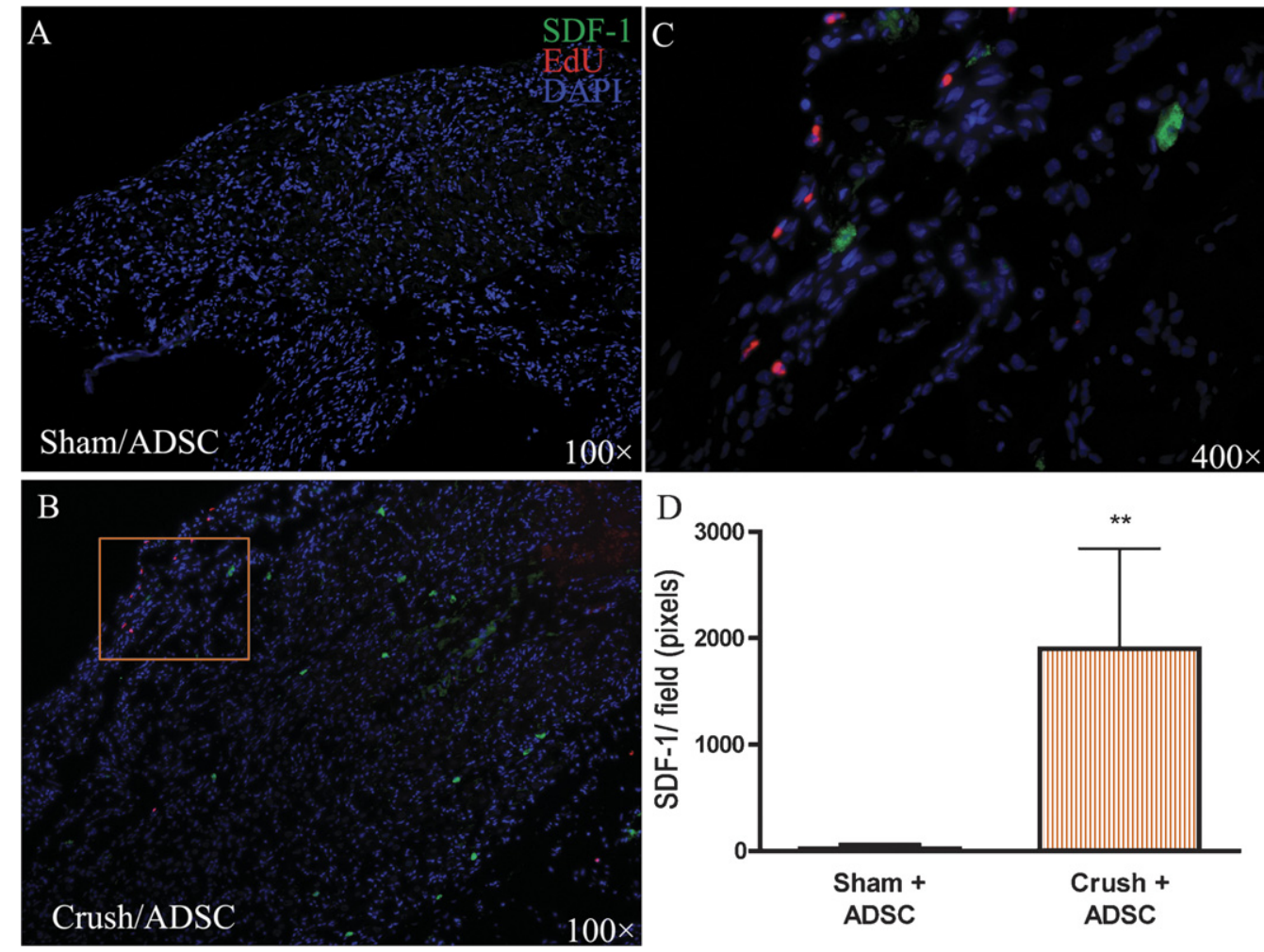

Fig. 6 - Detection of stromal cell-derived factor-1 (SDF-1) expression in the major pelvic ganglion. Rats ( $n=5$ in each group) were treated with sham operation or bilateral cavernous-nerve ( $\mathrm{CN}$ ) crush, and then intracavernously injected with 5-ethynyl-2-deoxyuridine (EdU)-labeled adipose-derived stem cells (ADSC). One day later, both EdU and SDF-1 were clearly visible in the major pelvic ganglia (MPG) of the (A) Crush + ADSC group, but not of the (B) Sham + ADSC group. (C) Higher magnification of the boxed area in panel B further shows the close association between SDF-1 expression and ADSC. Sections were stained for EdU, SDF-1, and 4',6-diamidino-2-phenylindole (DAPI). Original magnification: $\times 100$. (D) The graph demonstrates the expression levels of SDF-1 in the MPG $1 \mathrm{~d}$ after CN sham or crush injury. "p $<0.0001$ versus Sham + ADSC.
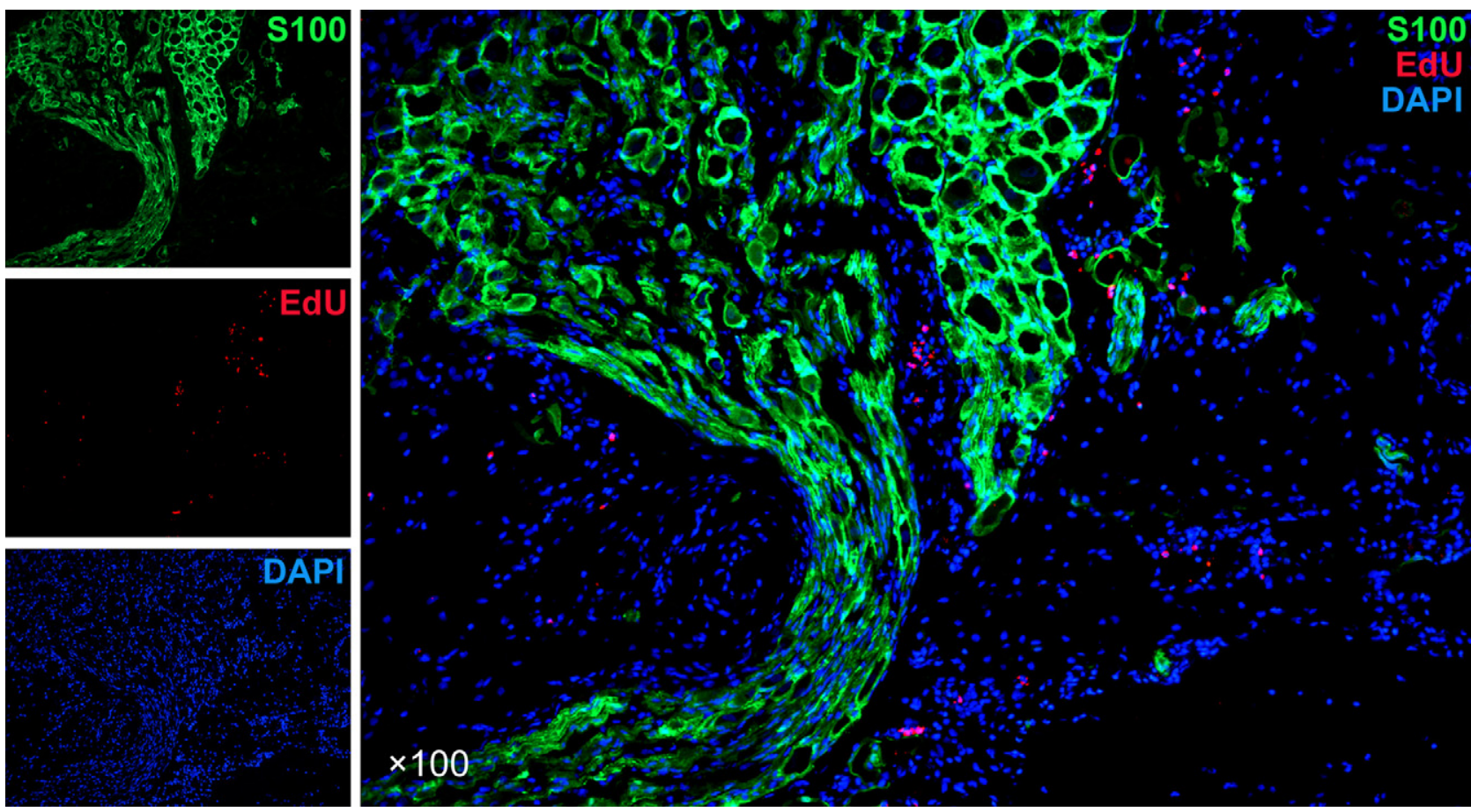

Fig. 7 - Presence of adipose-derived stem cells (ADSC) at the major pelvic ganglia $7 \mathrm{~d}$ after cavernous-nerve crush and intracavernous injection with 5-ethynyl-2-deoxyuridine (EdU)-labeled ADSC. S-100 protein was used to stain for nerve fibers, EdU staining for ADSC, and $4^{\prime}, 6^{\prime}$-diamidino-2phenyleindole (DAPI) for nuclear staining. Original magnification: $\times 100$. 
The study does have limitations. The observed changes were measured by immunofluorescence microscopy, which is semiquantitative. We reduced this limitation by performing all analyses in a computerized fashion. We observed short time-point changes only and are not aware of the effects of ADSC therapy in the long term. However, as nerve regeneration was observed, it is likely that this therapy is truly regenerative and thus long-term outcomes are putatively similar. Furthermore, we focused on one chemokine, based on previous literature and our own experience. While CXCR4 is known to play a role in cell recruitment, the full spectrum of chemokine receptors encompasses 21 molecules and it remains unknown what the role of these other receptors and other chemokines than SDF-1 may be. Further research into profiling chemokine secretion by nervous tissues and chemokine-receptor expression on ADSC is currently ongoing and could potentially identify additional goals to enhance ADSC recruitment, and thus efficacy.

\section{Conclusions}

These data, in conjunction with previous investigations, indicate that IC injected ADSC, and possibly other types of stem cells, exert their proerectile effects after $\mathrm{CN}$ injury by recruitment toward the MPG, where they initiate neural regeneration in the MPG.

Author contributions: Ching-Shwun Lin had full access to all the data in the study and takes responsibility for the integrity of the data and the accuracy of the data analysis.

Study concept and design: Fandel, Lue, Lin CS.

Acquisition of data: Fandel, Albersen, Lin G, Qiu, Ning, Banie.

Analysis and interpretation of data: Fandel, Albersen, Lin G, Lue, Lin CS.

Drafting of the manuscript: Fandel, Albersen, Lin CS.

Critical revision of the manuscript for important intellectual content: Fandel, Albersen, Lue, Lin CS.

Statistical analysis: Fandel.

Obtaining funding: Albersen, Lue.

Administrative, technical, or material support: Ning, Banie.

Supervision: Lue, Lin CS.

Other (specify): None.

Financial disclosures: I certify that all conflicts of interest, including specific financial interests and relationships and affiliations relevant to the subject matter or materials discussed in the manuscript (eg, employment/affiliation, grants or funding, consultancies, honoraria, stock ownership or options, expert testimony, royalties, or patents filed, received, or pending), are the following: $\mathrm{M}$. Albersen is a fellow of the Research Foundation - Flanders (FWO), and a scholar of the Federico Foundation. T.F. Lue is a consultant for Pfizer, Bayer, and Lilly; and recipient of a research grant from American Medical Systems. This work was supported by grants from the Arthur Rock Foundation and the US National Institutes of Health (DK045370).

Funding/Support and role of the sponsor: None.

\section{References}

[1] Heidenreich A, Bellmunt J, Bolla M, et al. EAU guidelines on prostate cancer. Part 1: screening, diagnosis, and treatment of clinically localised disease. Eur Urol 2011;59:61-71.
[2] Dubbelman YD, Dohle GR, Schröder FH. Sexual function before and after radical retropubic prostatectomy: a systematic review of prognostic indicators for a successful outcome. Eur Urol 2006; 50:711-20.

[3] Montorsi F, Brock G, Lee J, et al. Effect of nightly versus on-demand vardenafil on recovery of erectile function in men following bilateral nerve-sparing radical prostatectomy. Eur Urol 2008;54:924-31.

[4] Hatzimouratidis K, Burnett AL, Hatzichristou D, McCullough AR, Montorsi F, Mulhall JP. Phosphodiesterase type 5 inhibitors in postprostatectomy erectile dysfunction: a critical analysis of the basic science rationale and clinical application. Eur Urol 2009;55: 334-47.

[5] Bochinski D, Lin GT, Nunes L, et al. The effect of neural embryonic stem cell therapy in a rat model of cavernosal nerve injury. BJU Int 2004;94:904-9.

[6] Fall PA, Izikki M, Tu L, et al. Apoptosis and effects of intracavernous bone marrow cell injection in a rat model of postprostatectomy erectile dysfunction. Eur Urol 2009;56:716-26.

[7] Kendirci M, Trost L, Bakondi B, Whitney MJ, Hellstrom WJ, Spees JL. Transplantation of nonhematopoietic adult bone marrow stem/ progenitor cells isolated by p75 nerve growth factor receptor into the penis rescues erectile function in a rat model of cavernous nerve injury. J Urol 2010;184:1560-6.

[8] Lin G, Garcia M, Ning H, et al. Defining stem and progenitor cells within adipose tissue. Stem Cells Dev 2008;17:1053-63.

[9] Zuk PA. The adipose-derived stem cell: looking back and looking ahead. Mol Biol Cell 2010;21:1783-7.

[10] Albersen M, Fandel TM, Lin G, et al. Injections of adipose tissuederived stem cells and stem cell lysate improve recovery of erectile function in a rat model of cavernous nerve injury. J Sex Med 2010; 7:3331-40.

[11] Ma L, Hellstrom WJG. Re: treatment of erectile dysfunction in the obese type 2 diabetic ZDF rat with adipose tissue-derived stem cells. Eur Urol 2011;59:168-9.

[12] Lin G, Huang YC, Shindel AW, et al. Labeling and tracking of mesenchymal stromal cells with EdU. Cytotherapy 2009;11:864-73.

[13] Fandel TM, Bella AJ, Lin G, et al. Intracavernous growth differentiation factor-5 therapy enhances the recovery of erectile function in a rat model of cavernous nerve injury. J Sex Med 2008;5:1866-75.

[14] Albersen M, Fandel TM, Zhang H, et al. Pentoxifylline promotes recovery of erectile function in a rat model of postprostatectomy erectile dysfunction. Eur Urol 2011;59:286-96.

[15] Garcia MM, Fandel TM, Lin G, et al. Treatment of erectile dysfunction in the obese type 2 diabetic ZDF rat with adipose tissuederived stem cells. J Sex Med 2010;7:89-98.

[16] Huang YC, Ning H, Shindel AW, et al. The effect of intracavernous injection of adipose tissue-derived stem cells on hyperlipidemiaassociated erectile dysfunction in a rat model. J Sex Med 2010; 7:1391-400.

[17] Laurikainen A, Hiltunen JO, Vanhatalo S, Klinge E, Saarma M. Glial cell line-derived neurotrophic factor is expressed in penis of adult rat and retrogradely transported in penile parasympathetic and sensory nerves. Cell Tissue Res 2000;302:321-9.

[18] Dubovy P, Klusáková I, Svízenská I, Brázda V. Spatio-temporal changes of SDF1 and its CXCR4 receptor in the dorsal root ganglia following unilateral sciatic nerve injury as a model of neuropathic pain. Histochem Cell Biol 2010;133:323-37.

[19] Ahmadian Kia N, Bahrami AR, Ebrahimi M, et al. Comparative analysis of chemokine receptor's expression in mesenchymal stem cells derived from human bone marrow and adipose tissue. J Mol Neurosci 2011;44:178-85.

[20] George A, Buehl A, Sommer C. Wallerian degeneration after crush injury of rat sciatic nerve increases endo- and epineurial tumor necrosis factor-alpha protein. Neurosci Lett 2004;372:215-9. 
[21] Martini R, Fischer S, López-Vales R, David S. Interactions between Schwann cells and macrophages in injury and inherited demyelinating disease. Glia 2008;56:1566-77.

[22] Zhang H, Yang R, Wang Z, Lin G, Lue TF, Lin CS. Adipose tissuederived stem cells secrete CXCL5 cytokine with neurotrophic effects on cavernous nerve regeneration. J Sex Med 2011;8:437-46.
[23] Kalbermatten DF, Schaakxs D, Kingham PJ, Wiberg M. Neurotrophic activity of human adipose stem cells isolated from deep and superficial layers of abdominal fat. Cell Tissue Res 2011;344:251-60.

[24] Tan B, Luan Z, Wei X, et al. AMP-activated kinase mediates adipose stem cell-stimulated neuritogenesis of PC12 cells. Neuroscience 2011;181:40-7. 